\title{
Commercial Purity Titanium Processed by Rotary-Die Equal Channel Angular Pressing Method
}

\author{
Akira Watazu$^{1}$, Ichinori Shigematsu ${ }^{1}$, Aibin Ma ${ }^{1}$, Kazutaka Suzuki ${ }^{1}$, \\ Tsunemichi Imai ${ }^{1}$ and Naobumi Saito ${ }^{1}$ \\ ${ }^{1}$ National Institute of Advanced Industrial Science and Technology (AIST), \\ Materials Research Institute for Sustainable Development, Nagoya 463-8560, Japan
}

Commercial purity titanium cylindrical samples with a diameter of $11.5 \mathrm{~mm}$ and a length of $24 \mathrm{~mm}$ were processed under the condition of $773 \mathrm{~K}, 2.4 \mathrm{~mm} / \mathrm{s}$ punch, 1-4 equal channel angular pressing passes by a new severe plastic deformation process called the rotary-die equal channel angular pressing (RD-ECAP) process. Using this process, it was possible to conduct an ECAP process of 2 passes or over without sample removal, and the temperature of the commercial purity titanium could be controlled. Following the RD-ECAP process, the commercial purity titanium contained no cracks, and fine-grained microstructures were observed in the samples.

(Received April 26, 2005; Accepted July 29, 2005; Published September 15, 2005)

Keywords: commercial purity titanium, rotary-die equal channel angular pressing, fine grain, implant

\section{Introduction}

Commercial purity (cp) titanium is useful for biological applications such as dental, surgical and orthopedic implants, because cp-titanium can be fixed within a bone bed through direct attachment to living bone (osseointegration). ${ }^{1)}$ However, the mechanical properties of cp-titanium are not as high as those of some titanium alloys.

The production of fine-grained materials with high mechanical properties has been intensively studied using the severe plastic deformation method. ${ }^{2,3)}$ Recently, the equal channel angular pressing (ECAP) method invented by Segal et al. $^{4)}$ has proven successful for fabricating fine-grained bulk metals. By the ECAP method, a large strain can be introduced into a billet by simple shear deformation without changes in the cross-sectional area. In the ECAP process, the billet is extruded through a die consisting of two channels intersecting at an angle of $2 \Phi$. The sample is set in the vertical channel and pressed into the second channel. The greatest advantage of the ECAP method is that the initial size and shape of the sample processed by the ECAP process are maintained. However, in conventional ECAP, the pressed sample must be removed from the die and reinserted back for the next pressing, making the process inefficient. Therefore, it is difficult to control the temperature of the sample. Cptitanium samples processed by ECAP at room temperature which has not to be precisely controlled were studied by Semiatin et al. ${ }^{5)}$ The cp-titanium samples were fine-grained and had high mechanical strength, but the cp-titanium samples had cracks and thus split into pieces.

Recently, a new ECAP process method called rotary-die ECAP (RD-ECAP) ${ }^{6)}$ was developed by the author's group to form fine-grained bulk materials such as aluminium alloys, magnesium alloy and aluminium composites, ${ }^{7-12)}$ all of which can be processed under conditions of 573-673 K, at about a $0.9-2.4 \mathrm{~mm} / \mathrm{s}$ punch speed of $300 \mathrm{MPa}$ or lower. Schematic diagrams of the RD-ECAP are shown in Fig. 1. It consists of four channels meeting at the center of the rotary die and four punches in the corresponding channels. During the extrusion, two channels were blocked with their punches and the die holder wall as conventional ECAP process. After the extrusion, the die was rotated clockwise $90^{\circ}$ to the initial configuration and the second pressing was then performed. The die returns to its former position after 4 passes of the ECAP. By the RD-ECAP method, ECAP processing of up to 2 passes can be done without sample removal. Therefore, the temperature of the sample can be easily controlled. In our previous studies, ${ }^{6-11)}$ fine-grained aluminium alloys and magnesium alloys with a diameter of $20 \mathrm{~mm}$ were obtained by the RD-ECAP. However, in the case of titanium, a high temperature and high press load may be needed for severe plastic deformation of cp-titanium because of its high
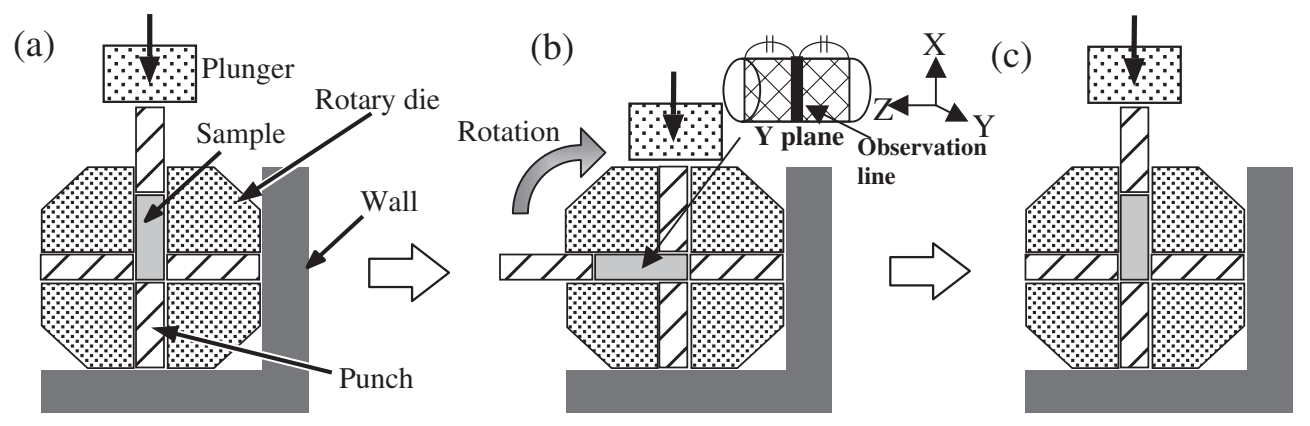

Fig. 1 Schematic diagram of rotary-die equal channel angular pressing. (a) initial state, (b) after one pass, and (c) after $90^{\circ}$ die rotation. 

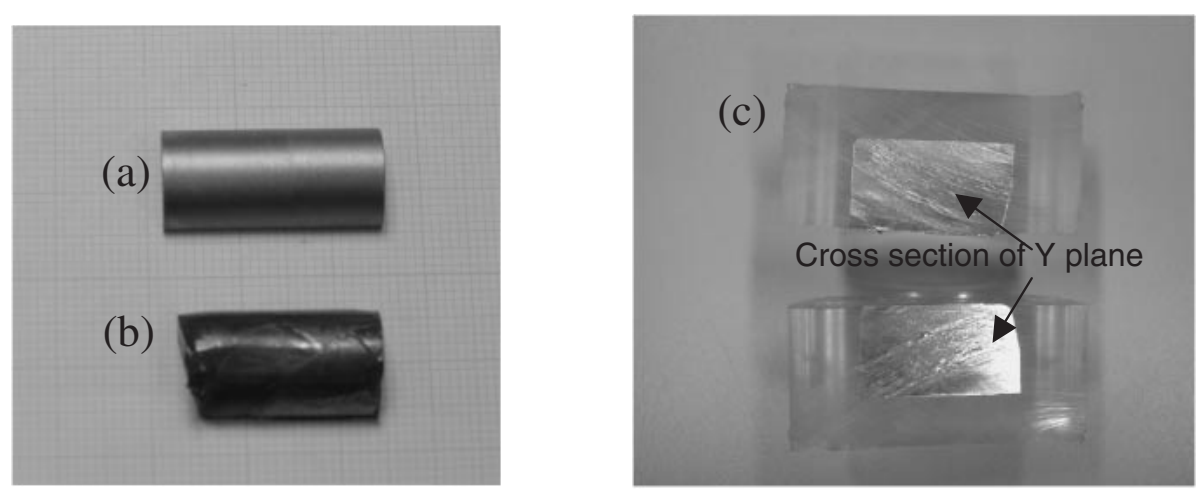

Fig. 2 Photograph of typical samples (a) before processing, (b) after 4 passes of RD-ECAP processing and (b) cross-section of Y plane.

mechanical strength. In this study, we report the RD-ECAP processing of cp-titanium with a diameter of $12 \mathrm{~mm}$.

\section{Experimental Procedures}

Cp-titanium of the JIS2 grade type was used as the raw material for the samples. Cylindrical samples with a diameter of $11.5 \mathrm{~mm}$ and a length of $24 \mathrm{~mm}$ were formed by lathing.

The sample and punches were put into the hole of the rotary die, which had a diameter of $12 \mathrm{~mm}$, and the die was set in a hot-press chamber. Then the die was heated to $773 \mathrm{~K}$ and the sample was pressed in the die. The punch speed when the sample was processed by RD-ECAP was about $2.4 \mathrm{~mm} / \mathrm{s}$ on average. The RD-ECAP processes were performed in air from 1 pass to 4 passes. After the RD-ECAP process was completed, the samples were cut and the microstructural characteristics of the RD-ECAP samples on the observation line of the Y plane were examined with an optical microscope after etching treatment.

\section{Results and Discussion}

The external appearances and Y plane of typical samples after 4 passes of RD-ECAP processing are shown in Fig. 2. After 4 passes of RD-ECAP, the samples had no cracks and had almost completely maintained their initial cylindrical shape. The surfaces of the cp-titanium samples were black. However, the cross-sections of the samples on the Y Plane were a metallic color. In our previous studies, the lubricant stuck to the surface of the samples and the surfaces of the samples also turned black. ${ }^{7,13)}$ In the cp-titanium in this study, the lubricant also stuck to the surface of the samples. Therefore, the material inside the samples did not become reactive, and the samples maintained a bulk of about $12 \mathrm{~mm}$ in diameter which did not crack and split apart. The press load for the cp-titanium deformation at 1 pass of RD-ECAP is shown in Fig. 3. The press load for the RD-ECAP processes of the cp-titanium samples were less than $60 \mathrm{kN}$. The maximum press load for 2-4 passes of RD-ECAP were also less than $60 \mathrm{kN}$. Consequently, cp-titanium with a diameter of $12 \mathrm{~mm}$ or over can be processed by the RD-ECAP method.

The typical Y plane microstructure of the cp-titanium samples before the RD-ECAP process are shown in Fig. 4. In the primary cp-titanium, grains over $30 \mu \mathrm{m}$ were observed. Figure 5 shows the optical micrographs representing the

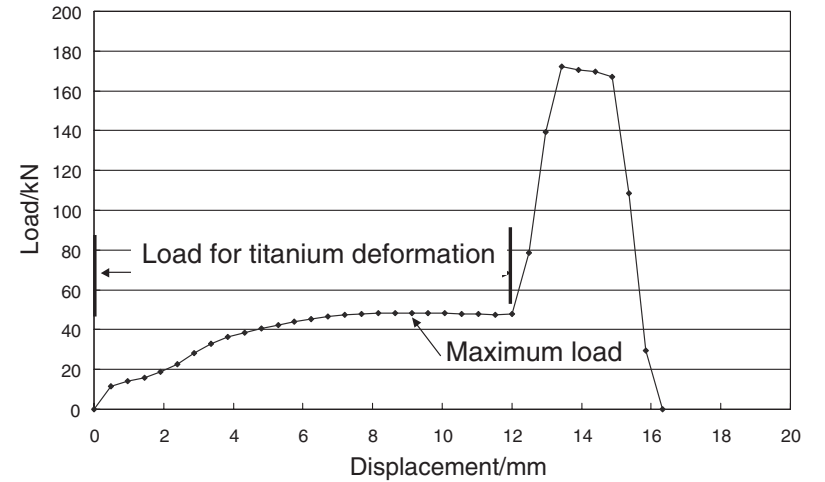

Fig. 3 Press load for cp-titanium deformation at 1 pass of RD-ECAP.

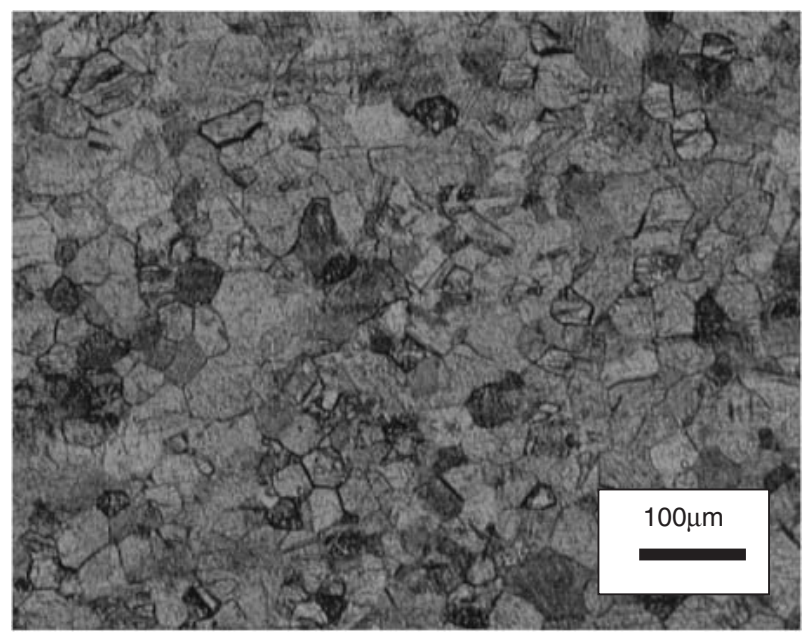

Fig. 4 The typical Y plane microstructure of the $\mathrm{cp}$ titanium before the RD-ECAP process.

typical fine-grained microstructure of the samples subjected to 1 pass RD-ECAP. The micrographs were taken from the center of the sample to the edge at every $0.75 \mathrm{~mm}$ on the observation line. Elongated fine grains can be observed in the sample. However, large grains like initial grains in the primary sample can be observed around the center area and in about a $2 \mathrm{~mm}$ area from the edge of the sample. Figure 6 shows the optical micrographs representing the typical finegrained microstructure of the samples subjected to 2 pass RD-ECAP. The sample had elongated fine grains around the center area. However, large grains like the initial grains 


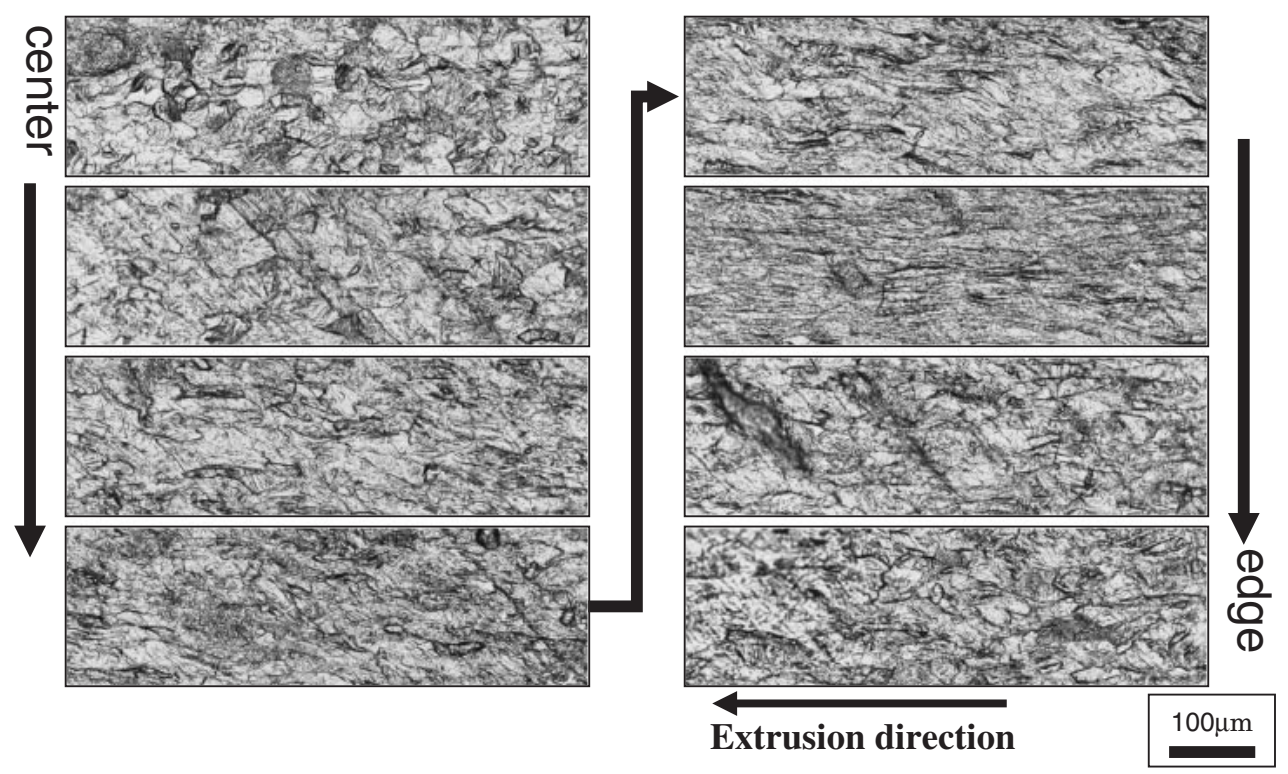

Fig. 5 Optical micrographs representing the typical fine-grained microstructure of the samples subjected to 1 pass RD-ECAP.
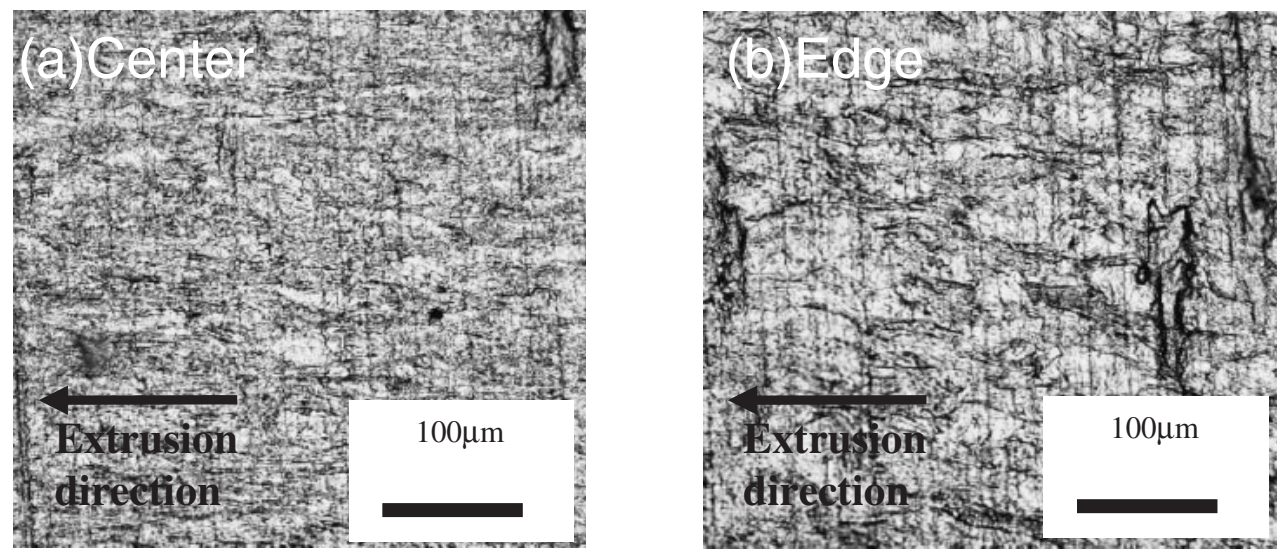

Fig. 6 Optical micrographs representing the typical fine-grained microstructure of the samples subjected to 2 pass RD-ECAP: (a) center area, (b) about $1 \mathrm{~mm}$ area from the edge.

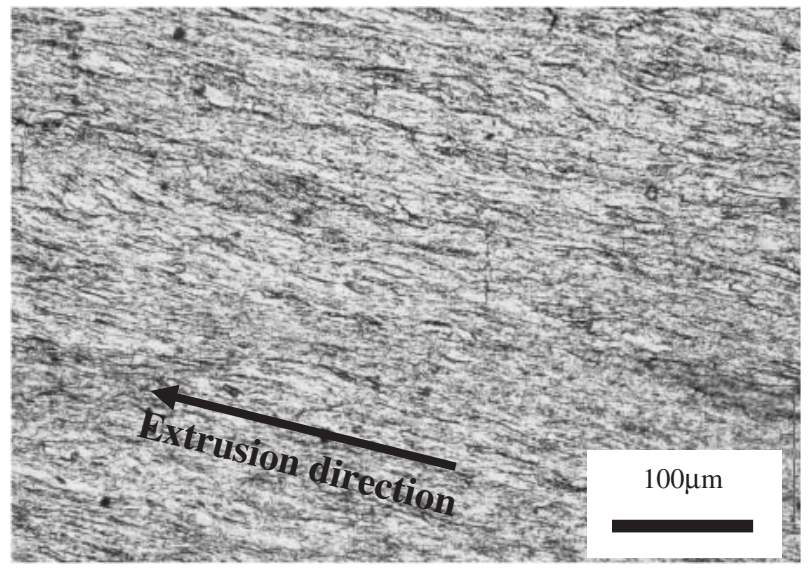

Fig. 7 Optical micrographs representing the typical fine-grained microstructure of the samples subjected to 3 pass RD-ECAP.

before RD-ECAP processing can be observed in about a $1 \mathrm{~mm}$ area from the edge of the sample. Figure 7 shows the optical micrographs representing the typical fine-grained microstructure of the samples subjected to 3 pass RD-ECAP.
The large grains in the sample decreased as compared with the sample processed by 2 passes of RD-ECAP. Figure 8 shows the optical micrographs representing the typical finegrained microstructure of the samples subjected to 4 pass RD-ECAP. Large grains like the initial grains before RDECAP processing can hardly be observed in the sample. Instead, elongated fine grains less than $2-3 \mu \mathrm{m}$ can be observed. In a previous study, ${ }^{5)}$ elongated fine grains of the order of micrometer were observed in cp-titanium samples processed by ECAP and the samples had high mechanical properties such as mechanical strength. Therefore, the samples processed by RD-ECAP also had elongated fine grains and expected to have high mechanical properties.

\section{Summary}

The RD-ECAP method was applied to cp-titanium in order to produce a fine-grained microstructure in cylindrical samples with a diameter of $11.5 \mathrm{~mm}$ and a length of $24 \mathrm{~mm}$. By the RD-ECAP, ECAP processes of 1-4 passes were possible without sample removal and the temperature of 

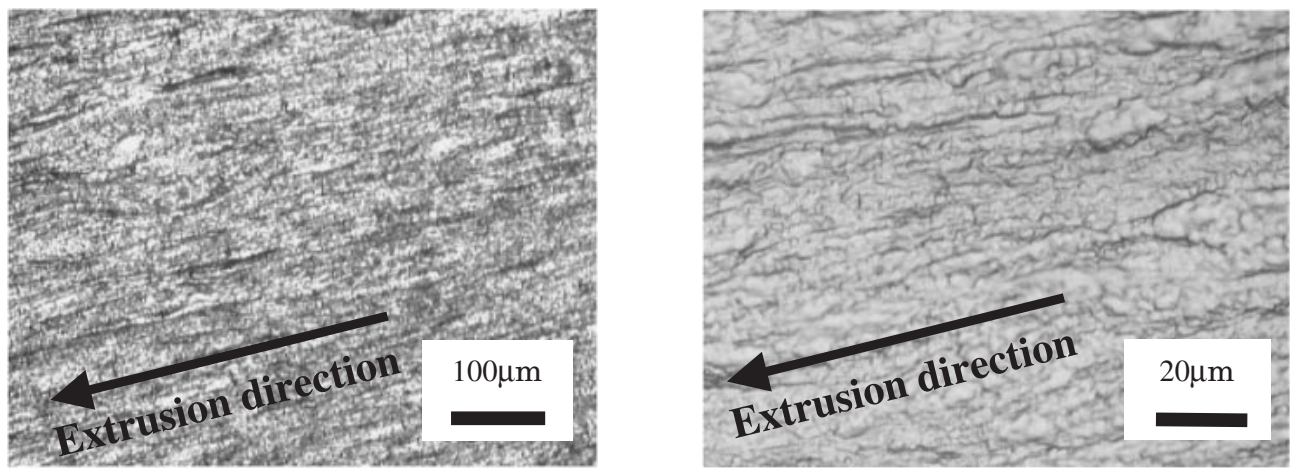

Fig. 8 Optical micrographs representing the typical fine-grained microstructure of the samples subjected to 4 pass RD-ECAP.

the cp-titanium could be easily controlled. After the RDECAP process of 1-4 passes, the cp-titanium samples had no cracks and fine-grained microstructures were observed. In the sample processed by 4 passes, large grains like the initial grains before RD-ECAP were hardly observed. Therefore, the cp-titanium processed by RD-ECAP is expected to have high mechanical properties.

\section{REFERENCES}

1) P.-I. Bränemark: J. Prosthet. Dent. 50 (1983) 399-410.

2) R. Z. Valiev, A. V. Korznikove and R. R. Mulyukov: Mater. Sci. Eng. A 168 (1993) 141-148.

3) R. Z. Valiev, R. R. Mulyukov, V. V. Ovchinnikove and V. A. Shabashov: Scr. Metall. Mater. 25 (1991) 2717-2722.

4) V. M. Segal, V. I. Reznikov, A. E. Drobysevsky and V. I. Kopylov: Metally 1 (1981) 99-105.
5) S. L. Semiatin, V. M. Segal, R. E. Goforth, N. D. Frey and D. P. Delo: Metall. Mater. Trans. A 30A (1999) 1425-1435.

6) Y. Nishida, H. Arima, J. C. Kim and T. Ando: J. Jpn. Inst. Light Met. 64 (2000) 655-659.

7) Y. Nishida, H. Arima, J. C. Kim and T. Ando: J. Jpn. Inst. Met. 64 (2000) 1224-1229.

8) Y. Nishida, H. Arima, J. C. Kim and T. Ando: Scr. Mater. 45 (2001) 261-266.

9) A. Ma, Y. Nishida, I. Shigematsu, N. Saito, A. Watazu, T. Ando, S. Lim and M. Nagase: Proc. International Symposium on Superplasticity and Superplastic Forming Technology 20021 (2003) 44-50.

10) A. Ma, Y. Nishida, N. Saito, I. Shigematsu and S.-W. Lim: Mater. Sci. Tech. 19 (2003) 1642-1647.

11) A. Ma, N. Saito, I. Shigematsu, K. Suzuki, M. Takagi, Y. Nishida, H. Iwata and T. Imura: Mater. Trans. 45 (2004) 399-402.

12) A. Ma, K. Suzuki, Y. Nishida, N. Saito, I. Shigematsu, M. Takagi, H. Iwata, A. Watazu and T. Imura: Acta Mater. 53 (2005) 211-220.

13) A. Watazu, Y. Nishida, I. Shigematsu and J. Zhu: Proc. 10th World Conference on Titanium 1 (2004) 343-346. 